\title{
Standoff Methods for the Detection of Threat Agents: A Review of Several Promising Laser-Based Techniques
}

\author{
J. Bruce Johnson, ${ }^{1}$ Susan D. Allen, ${ }^{2}$ Jonathan Merten, ${ }^{1}$ \\ Lewis Johnson, ${ }^{3}$ Daniel Pinkham, ${ }^{4}$ and Scott W. Reeve ${ }^{1}$ \\ ${ }^{1}$ Arkansas Center for Laser Applications and Science, Arkansas State University, Jonesboro, AR 72401, USA \\ ${ }^{2}$ Department of Mechanical Engineering, Embry Riddle Aeronautical University, Daytona Beach, FL 32114, USA \\ ${ }^{3}$ Department of Physics, Florida A\&M University, Tallahassee, FL 32310, USA \\ ${ }^{4}$ U.S. Army RDECOM CERDEC, Night Vision \& Electronic Sensors Directorate, Fort Belvoir, VA 22060, USA
}

Correspondence should be addressed to Scott W. Reeve; sreeve@astate.edu

Received 14 February 2014; Accepted 22 May 2014; Published 7 August 2014

Academic Editor: Eugen Culea

\begin{abstract}
Copyright (C) $2014 \mathrm{~J}$. Bruce Johnson et al. This is an open access article distributed under the Creative Commons Attribution License, which permits unrestricted use, distribution, and reproduction in any medium, provided the original work is properly cited.

Detection of explosives, explosive precursors, or other threat agents presents a number of technological challenges for optical sensing methods. Certainly detecting trace levels of threat agents against a complex background is chief among these challenges; however, the related issues of multiple target distances (from standoff to proximity) and sampling time scales (from passive mines to rapid rate of march convoy protection) for different applications make it unlikely that a single technique will be ideal for all sensing situations. A number of methods for spanning the range of optical sensor technologies exist which, when integrated, could produce a fused sensor system possessing a high level of sensitivity to threat agents and a moderate standoff real-time capability appropriate for portal screening of personnel or vehicles. In this work, we focus on several promising, and potentially synergistic, laser-based methods for sensing threat agents. For each method, we have briefly outlined the technique and report on the current level of capability.
\end{abstract}

\section{Introduction}

Real time detection of explosives and explosive devices continues to represent a major challenge for US and allied military forces in conflict areas throughout the world. Over the last decade or so, there have been a number of review articles that have addressed this subject from a variety of perspectives [1-6]. One of the clear outcomes from this body of work is that the ability to deter, predict, detect, respond to, and mitigate both improvised as well as conventional explosive threats is an issue from both a military and homeland security perspective. Explosives represent only one area of concern, as the same sensing technology could be easily adaptable to the general class of threat agents that includes nuclear, biological, and environmental toxins.

The ideal detection system for threat agents, whether these threats are explosives, chemical and biological agents, or illicit drugs, would possess high sensitivity (i.e., be capable of detecting threats present only at trace levels), be able to discern a threat amidst a real world background, possess a standoff capability, be straightforward to operate, and possess a real time sensing potential [1-19]. This last operational criterion will be critical whether the sensor is deployed at a military checkpoint, for airport security, or for any other domestic application where impeding the flow of commerce is an issue. Although such an ideal sensor system does not currently exist, there are a number of optically based techniques that, individually, possess one or more of these operational characteristics [1-23].

Consider that threat signatures are likely to come in two broad categories. Using explosives as an example, low vapor pressure solid and liquid residue on an automobile door or door handle left behind by someone who has recently handled explosives represents one type of signature. The second broad class of threat signatures consists of vapor emissions due to volatile solvents, impurities, and decomposition products 
naturally occurring in explosive material. These explosive related volatile organic compounds (VOCs) are collectively referred to as "the explosive bouquet" and are believed to represent the same signature used by explosive-trained canines for sensing the presence of an explosive [7-19, 24-27]. Also included in this vapor signature category is the notion of a "breath test" to determine exogenous chemicals in exhaled breath [28]. Exogenous chemical spot breath measurement methods are already widely used within some governmental agencies and/or law enforcement communities. Evidentiary breath alcohol determination and EPA environmental exposure assessment studies represent two such examples [28, 29].

From a sensing perspective, individuals who have handled or have been exposed to threat agents (inhalation, ingestion, and dermal exposures are common routes) that contain a mixture of VOCs (such as a TNT-based explosive) will exhale these compounds for some time after exposure. Indeed, there have been a number of studies investigating the time profile for breath elimination of VOCs $[28,29]$. Less volatile threat agents are often metabolized, ultimately leading to the production of volatile biomarker compounds. These biomarkers are typically quite sensitive and specific to a particular agent. Moreover, the volatile biomarkers cannot be disguised from an appropriate analytical measurement any more than an impaired driver can disguise the amount of alcohol in their breath.

Detection of threat agents will necessarily require a sensor able to monitor both particle and vapor signatures simultaneously. Again, there are several optical techniques which, when combined, could potentially produce an integrated sensor system possessing a high sensitivity towards threat agents as well as the ability to discriminate these threat species from a complex background signal at moderate standoff distances $(1 \mathrm{~m}<x<10 \mathrm{~m})$ and in real time. For example, cavity ringdown laser absorption (CRD or CRDLAS) spectroscopy has demonstrated a great deal of potential for sensing at short $(<1 \mathrm{~m})$ distances. Indeed, there have been a number of review articles, books, and treatises that have focused on this powerful spectroscopic technique $[20,21]$. There have also been a number of reviews that have focused on Raman scattering and laser-induced-breakdown spectroscopic (LIBS) methods for standoff sensing applications $[1-6,22]$.

Within the Arkansas Center for Laser Applications and Science (ArCLAS), we have created a number of unique sensing capabilities over the last decade. Here we examine the performance of several of these sensor systems with a potential standoff threat detection capability in some detail, including an enhanced LIBS method, in which a collinear $\mathrm{CO}_{2}$ TEA laser pulse is superimposed in time and space with the tripled or quadrupled output from an Nd:YAG laser as well as an enhanced Raman-based method known as stimulated Raman adiabatic passage or STIRAP [30]. In addition to the spectroscopic sensing capability, we have also developed a capability to generate molecular signatures for threat agents of interest. We will begin with a brief discussion of the molecular signature effort by examining several examples relevant to explosive detection.

\section{Molecular Signatures}

Every optically based sensing method requires a priori knowledge of the molecular signature, or in this case, wavelength specific signature, for the species of interest. For many species, high quality, low spectral resolution $\left(\sim 0.1 \mathrm{~cm}^{-1}\right)$ databases are sufficient. There are literally person-decades of effort contained in these infrared databases [31]. And, for many small molecules, spectral resolution of this magnitude is sufficient for achieving rotationally resolved vibrational spectra. When greater specificity or discrimination is required, high spectral resolution signatures are needed. Fortunately, there are also a number of high quality, high spectral resolution databases that have been developed and maintained. The HITRAN database is one example of such a database [32, 33]. However, many of these high-resolution databases contain spectral information for a limited number of species. For example, although the current HITRAN database contains over 7 million rotationally resolved transitions, only 47 different molecular species (and their isotopic variations) are represented $[32,33]$. Nevertheless, most of the major species relevant for atmospheric analysis are contained in this high resolution spectral database.

In the case of explosives, it is often not the energetic compound that is of interest, but rather a volatile impurity or perhaps a photo fragment. For TNT-based explosives the volatile explosive bouquet consists of toluene, nitrobenzene, and the mononitrotoluene (MNT) [34]. A detailed database containing the high resolution spectral signature for such compounds is extremely limited, in part due to the fact that each is an asymmetric top and is expected to exhibit fairly complex spectra when examined at high spectral resolution and room temperature conditions.

For many bouquet species, again, high quality low resolution infrared data is widely available [35-46]. To a significant extent, we have used these databases as a starting point for our high resolution infrared measurements. All of the infrared measurements shown here were collected using the spectrometers shown in Figure 1 (a detailed description of the spectrometer is available in the literature $[47,48])$. For the longer wavelength measurements, various $\mathrm{Pb}$-salt diode lasers were utilized as the primary light source. At shorter wavelengths, a combination of $\mathrm{Pb}$-salt diode lasers and $\mathrm{cw}$ external cavity quantum cascade lasers (QCLs) was used.

Consider the snippet of rovibrational spectrum for nitrobenzene shown in Figure 2. This particular trace was recorded at a spectral resolution of $0.003 \mathrm{~cm}^{-1}$ with a $\mathrm{Pb}$ salt diode laser. The top trace represents the signal channel and contains the spectral data for nitrobenzene. The middle trace is from a $1^{\prime \prime}$ solid Ge etalon and is used for calibration purposes. The bottom panel contains a reference gas used to provide absolute frequency calibration of signal. In this spectral region, $\mathrm{CO}_{2}$ is often used as a reference gas and the trace shows a series of well-known rovibrational transitions for the various isotopic varieties of $\mathrm{CO}_{2}$. Although this snippet is less than $2 \mathrm{~cm}^{-1}$ in frequency space, there are 17 clearly defined nitrobenzene absorptions. To date, dozens of these nitrobenzene snippets have been observed and 


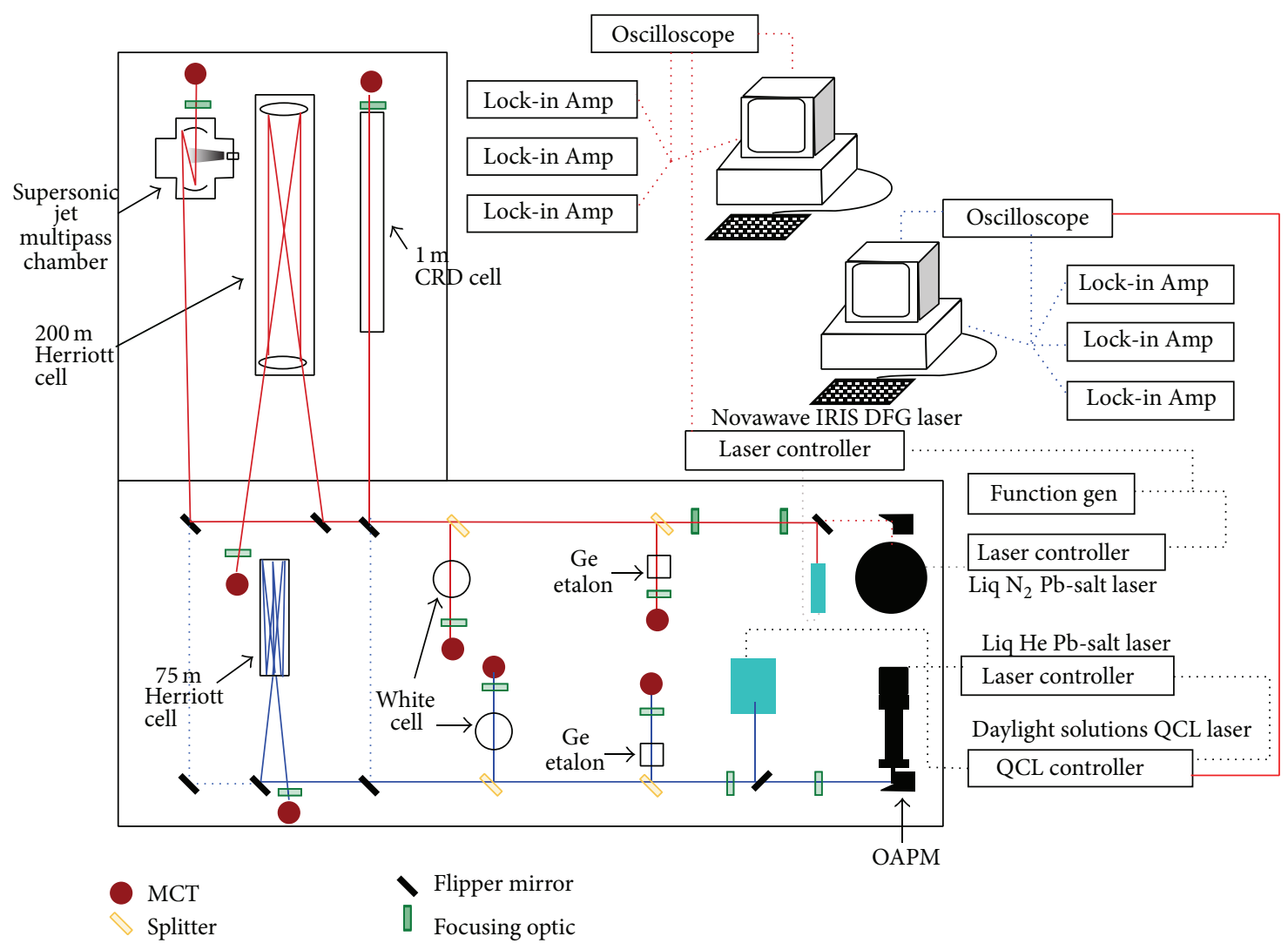

FIGURE 1: ArCLAS infrared system for determining spectroscopic signatures. There are numerous infrared light sources available including $\mathrm{Pb}$-salt diode lasers, a Novawave DFG laser designed for operation near 3 microns, and four Daylight Solutions cw QCL lasers designed for operation at $10.6,7.5,5.5$, and 4.5 microns, respectively.

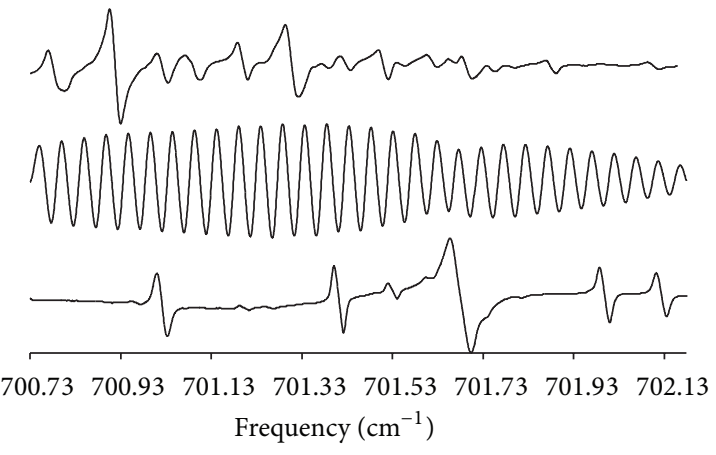

FIGURE 2: A representative spectral snippet from the nitrobenzene spectrum in the 14-micron region. This trace was recorded utilizing a $\mathrm{Pb}$-salt diode laser spectrometer and a FM modulated detection scheme. There are 17 clearly defined rotationally resolved nitrobenzene transitions FM modulated signal shown in the top panel. The lower traces are recorded simultaneously with signal channel and are used for frequency calibration purposes.

hundreds of rotationally resolved nitrobenzene transitions have been recorded and tabulated in the 14-micron region.

While a comprehensive table of nitrobenzene rovibrational absorptions near $700 \mathrm{~cm}^{-1}$ has been generated, we have not been successful assigning a complete set of rotational quantum numbers to these transitions. Certainly, the complex nature of the spectra contributes to the challenging nature of generating accurate assignments. Another contributing aspect however is the discrete tuning limitation associated with $\mathrm{Pb}$-salt diode lasers and this limitation is particularly true at longer wavelengths where $\mathrm{He}$ cooled cryogenic cooling is necessary for laser operation. Continuous tuning is accomplished over a single longitudinal mode of the laser. Helium cooled diodes typically possess very short longitudinal mode tuning ranges in frequency space $\left(<1 \mathrm{~cm}^{-1}\right.$ in many cases) with adjacent modes being separated by $3-5 \mathrm{~cm}^{-1}$.

The molecular signatures for species associated with RDX-based explosives do not typically contain nitro groups $[32,33,49]$. In fact, based on a series of GC/MS measurements $[32,33,49]$, the explosive bouquet for RDX-based contains cyclohexanone, ethylhexanol, and isobutylene. Of these compounds, isobutylene lends itself to high resolution spectroscopic study most readily. Several rovibrational bands of isobutylene occur at shorter wavelengths and these bands have been successfully investigated with $\mathrm{Pb}$-salt diode laser technology. For example, the $v_{28}$ band at 11 microns has been observed, analyzed, and assigned, at least for the torsion-free vibrational bands [50]. Some headway has also been achieved on the $v_{7}$ band centered near $1380 \mathrm{~cm}^{-1}$ [51]. What is interesting about these two bands is the observed regularity associated with the $\mathrm{P}, \mathrm{Q}$, and $\mathrm{R}$ branches.

Consider the FTIR spectrum for this band shown in Figure 3. This particular band was recorded at a spectral resolution of only $0.125 \mathrm{~cm}^{-1}$. Even so, there appears to be a 


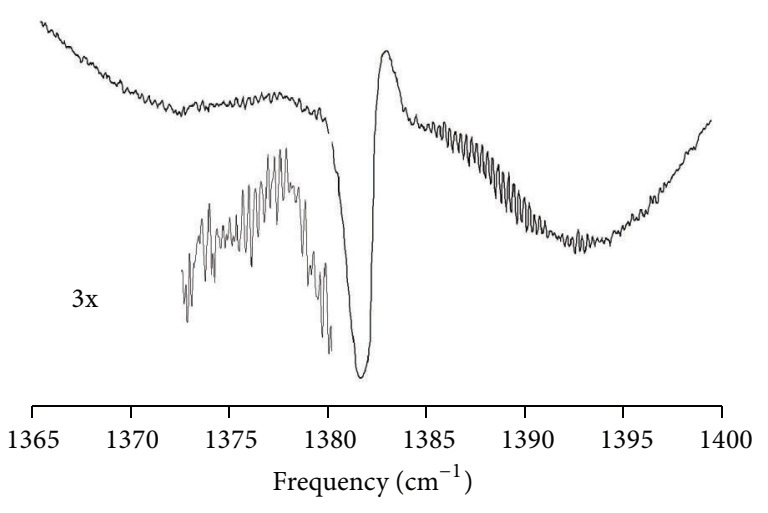

FIGURE 3: Low resolution FTIR spectrum of the $v_{7}$ of isobutylene.

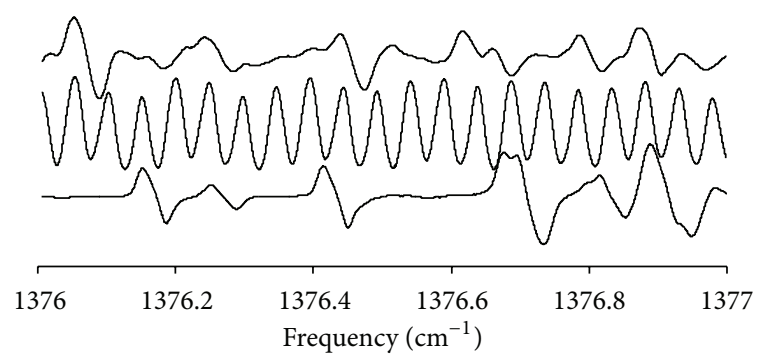

FIGURE 4: High resolution scan obtained with an isobutylene vapor sample at a total pressure of 80 mTorr in a $75 \mathrm{~m}$ Herriott cell. The top trace is the signal channel containing the isobutylene sample. The middle trace is an interference pattern generated by a solid Ge etalon. The last trace is due to methyl chloride. Again the bottom two traces are collected for calibration purposes with the etalon being used to transform the time-based signal into $\mathrm{cm}^{-1} /$ point and the reference gas channel providing an absolute position in frequency space.

series of rotationally resolved transitions in both the $\mathrm{P}$ and $\mathrm{R}$ branches of the band.

At higher spectral resolution, it becomes clear that there is additional structure associated with each spectral feature in the P and R branches. In Figure 4, a spectral snippet from the same region taken with $\mathrm{Pb}$ salt diode laser is shown. In the top panel of Figure 4, three sets of doublets are observed in the isobutylene signal. The spacing of the doublets is approximately $0.5 \mathrm{~cm}^{-1}$ (from doublet center to doublet center). A similar spacing is observed in the lower resolution FTIR spectrum in Figure 3 and corresponds to the spacing between adjacent spectral features in the $\mathrm{P}$ and $\mathrm{R}$ branches.

Rotational $J$ assignments have been tentatively made using a spherical top type model and, with these assignments in hand, a series of effective molecular constants were determined for the $v_{7}$ band [51]. A complete listing of these constants is given in Table 1.

Rotational constants for the ground vibrational state of isobutylene were originally determined using microwave spectroscopic methods and first reported over forty years ago [52]. The set of rotational constants determined from the microwave measurements are $A_{0}=0.304654 \mathrm{~cm}^{-1} ; B_{0}=$ $0.279587 \mathrm{~cm}^{-1}$; and $C_{0}=0.153972 \mathrm{~cm}^{-1}$ [52]. Unfortunately,
TABLE 1: Effective molecular constants for the $v_{7}$ band of isobutylene*.

\begin{tabular}{lc}
\hline$v_{0}$ & $1381.49(15)$ \\
$B_{1}$ & $0.225(6)$ \\
$D_{1}$ & $4.7(8) \times 10^{-5}$ \\
$B_{0}$ & $0.224(6)$ \\
$D_{0}$ & $4.5(7) \times 10^{-5}$ \\
\hline${ }^{*}$ All values are in $\mathrm{cm}^{-1}$. &
\end{tabular}

there is really no direct way to correlate the effective $B_{0}$ rotational constant in Table 1 with the rotational constants reported by Laurie and coworkers. Such correlations can really only be obtained by fitting the rovibrational data to an oblate asymmetric top set of energy expressions. Although the goal for any high resolution molecular spectroscopic study should necessarily include such correlations, the central point of this paper is to highlight a strategy to identify appropriate spectral regions for optical detection of explosive bouquet species. Parenthetically, we are working to fit the rovibrational data for the $1382 \mathrm{~cm}^{-1}$ band of isobutylene so that these correlations can be made.

In fact, this band has also been examined with a Daylight Solutions mode-hop-free QCL laser spectrometer. The entire $v_{7}$ band has been subsequently recorded at spectral resolutions ranging from 0.1 to $0.001 \mathrm{~cm}^{-1}$ [53]. A detailed analysis, using a more appropriate asymmetric top model, has been performed resulting in a unique $J, K_{a}, K_{c}$ quantum assignment for each of the observed spectral transitions [53].

\section{Stimulated Raman Adiabatic Passage (STIRAP)}

Laser induced fluorescence is a very sensitive technique for monitoring molecular species. Unfortunately, it is not very useful for many of the species of interest for threat detection in the atmosphere. These relatively small molecules frequently require excitation in the ultraviolet and there are many other trace species in the atmosphere that are also excited in the uv, significantly complicating result interpretation. Techniques that use two photons for excitation allow much more selectivity in targeting the molecule of interest but at a cost of more instrument complexity. One such technique is Stimulated Raman adiabatic Passage or STIRAP.

STIRAP was developed and first described by Gaubatz et al. [54]. A brief outline of this method is given here. In its most basic form, STIRAP requires three energy levels of an atom or molecule with good electric dipole coupling between an initial state $|1\rangle$ and an intermediate state $|2\rangle$ as well as good electric dipole coupling between the intermediate state and a final state $|3\rangle$ (see Figure 5).

In order to perform ordinary sequential excitation or stimulated emission pumping (SEP) the first laser (pump) excites the molecule from level $|1\rangle$ to level $|2\rangle$ after which the second laser (Stokes) excites from level $|2\rangle$ to level $|3\rangle$. STIRAP differs in that the order of the laser pulses is reversed, albeit with partial temporal overlap. This counterintuitive 


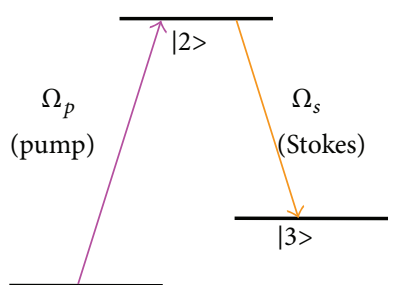

|1>

Resonance

$\wedge$ scheme

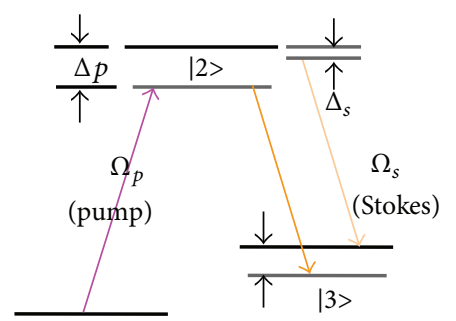

$\mid 1>$

$$
\begin{gathered}
\text { Off-resonance } \\
\wedge \text { scheme }
\end{gathered}
$$

FIGURE 5: Energy-level diagram of three states in a molecule or atom. $\omega_{p}$ represents the angular frequency of the pump photons which couple states $|1\rangle$ and $|2\rangle . \omega_{s}$ represents the angular frequency of the Stokes photons which couple states $|2\rangle$ and $|3\rangle$. In the offresonance diagram the photon energies are detuned by an amount $\Delta_{p}$ for the pump photons, and $\Delta_{s}$ for the Stokes photons yielding a two-photon detuning of $\delta=\Delta_{p}-\Delta_{s}$ for the combination. The heavy black horizontal lines correspond to energy states in the atom or molecule. The grey lines, which are present for illustration only, do not correspond to energy states.

sequence is capable of transferring $100 \%$ of the atoms or molecules from the initial state to the final state, whereas SEP transfers a smaller fraction to the final state (much smaller when weaker laser pulses are used). STIRAP can be performed with any ordering of the atomic/molecular energy levels with lambda, $\Lambda$, (initial and final states lower than the intermediate state) and ladder (final state higher than the intermediate state which is higher than the initial state) being the most common.

Diagrams outlining the STIRAP process in the lambda ordering of energy levels are shown in Figure 5. In the panel on the left the wavelengths of the lasers are tuned to correspond precisely to the differences in the energy levels of the atom or molecule and efficient transfer from state $|1\rangle$ to state $|3\rangle$ can occur. This is the desired arrangement for the target molecule. In the panel on the right (off-resonance), the energy/photon of light coupling states $|1\rangle$ and $|2\rangle\left(\hbar \omega_{p}\right)$ is less than the difference in energy between $|1\rangle$ and $|2\rangle$ by an amount $\Delta_{p}$. Likewise, the energy/photon of light coupling states $|2\rangle$ and $|3\rangle\left(\hbar \omega_{s}\right)$ is less than the difference in energy between states $|2\rangle$ and $|3\rangle$ by an amount $\Delta_{s}$. The total mismatch for the two-photon transition from $|1\rangle$ to $|3\rangle$ is $\delta=\Delta_{p}-\Delta_{s}$.

Within the rotating-wave approximation, the quantum mechanics of a three-state system can be modeled by the following set of equations:

$$
i\left[\begin{array}{c}
\dot{C}_{1} \\
\dot{C}_{2} \\
\dot{C}_{3}
\end{array}\right]=\left[\begin{array}{ccc}
0 & \frac{\Omega_{p}(t)}{2} & 0 \\
\frac{\Omega_{p}(t)}{2} & \Delta_{p} & \frac{\Omega_{s}(t)}{2} \\
0 & \frac{\Omega_{s}(t)}{2} & \delta
\end{array}\right]\left[\begin{array}{c}
C_{1} \\
C_{2} \\
C_{3}
\end{array}\right]
$$

where $\left|C_{1}\right|^{2},\left|C_{2}\right|^{2}$, and $\left|C_{3}\right|^{2}$ represent the populations of the states $|1\rangle,|2\rangle$, and $|3\rangle ; \dot{C}_{1}, \dot{C}_{2}$, and $\dot{C}_{3}$, represent time derivatives of $C_{1}, C_{2}$, and $C_{3}$; and $\Omega_{p}(t)$ and $\Omega_{s}(t)$ represent the time dependent Rabi (angular) frequencies of the laser pulses. The solution to (1) with the pump and Stokes laser pulses of Gaussian temporal shape shown in Figure 5 is shown in Figure 6(b). The population of state $|3\rangle$ starts at zero and increases to 1 while the pump and Stokes pulses overlap. When the pulses have passed, the population of state $|3\rangle$ differs negligibly from unity. It is interesting to note that the state $|2\rangle$ remains relatively unpopulated. This is an advantage since state $|2\rangle$ can fluoresce which could lead to a decrease in the population transfer efficiency to state $|3\rangle$. The results shown in Figure 6 have been reproduced experimentally on a number of atomic and molecular species [54-57].

One of the advantages of using STIRAP to study molecules in the atmosphere is that the method is robust with respect to variations in the timing of the pulses. Figure 7 shows the fraction of molecules transferred to state $|3\rangle$ as a function of the temporal delay of the pump pulse behind the Stokes pulse. In this solution to (1) the integrated Rabi frequencies of the laser pulses was set equal to 20 radians and the pulse widths of the lasers were arbitrarily set to $62 \mathrm{ps}$. The transfer efficiency remains above $90 \%$ for delays between 31 and 82 ps (a range greater than $80 \%$ of the pulse width). While the width of the pulses in this calculation is arbitrary, the result of $80 \%$ is not. When the integrated Rabi frequencies of the laser pulses is 20 radians, the transfer efficiency to state $|3\rangle$ remains above $90 \%$ for a range of greater than $80 \%$ of the laser pulse width, independent of the laser pulse width. The robustness of the STIRAP transfer efficiency with respect to laser pulse timing delay is ideal for propagation through the atmosphere; atmospheric dispersion could potentially change the delay of one pulse relative to another. Although the dispersion could be incorporated into the delay between the pulses for measurements at a certain distance, the robustness of the method with respect to this delay makes measurements at a larger range of distances possible.

The vast majority of published STIRAP papers have been done on atomic/molecular beams or ultracold gases. These sample arrangements eliminate collisions and Doppler broadening which makes it possible to use continuous wave lasers or Fourier-transform-limited nanosecond lasers. Inasmuch as collisions between the target atom/molecule with other atoms or molecules spoil the STIRAP transfer efficiency, when collisions are present the STIRAP transfer must be complete in a time shorter than the mean time between collisions. If STIRAP is to be performed at atmospheric pressure, the process must be complete in a time shorter than a few tens of picoseconds.

In order to demonstrate STIRAP on a vapor (as opposed to an atomic beam or ultracold gas), we performed STIRAP on a vapor of sodium using coherent lasers with pulse widths of several picoseconds. The initial, intermediate, and final states were chosen to be the $3 \mathrm{~s}, 3 \mathrm{p}\left({ }^{2} \mathrm{P}_{3 / 2}\right)$, and $5 \mathrm{~s}$ states, respectively. Figure 8 shows STIRAP in sodium. The population transfer efficiency in the STIRAP ordering of laser pulses is about a factor of two larger than the SEP ordering. In a paper to be published, we show that with strong laser pulses 


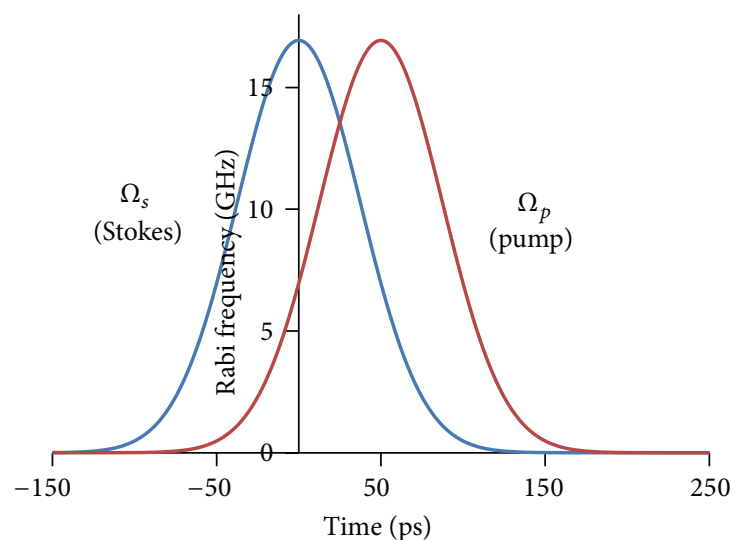

(a)

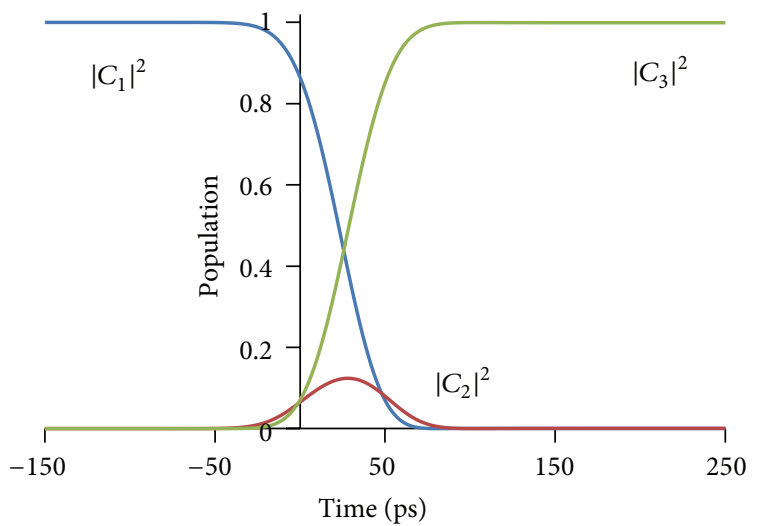

(b)

Figure 6: Rabi frequencies and population transfers as a function of time. (a) The Rabi frequencies of the pump and Stokes laser pulses. Note that the Stokes pulse (coupling states $|2\rangle$ and $|3\rangle$ ) precedes the pump pulse (coupling states $|1\rangle$ and $|2\rangle$ ). (b) The populations of states $|1\rangle$, $|2\rangle$, and $|3\rangle$ as a function of time. Note that the population shifts from $|1\rangle$ to $|3\rangle$ during the time when the laser pulses overlap with very little population ever reaching state $|2\rangle$.

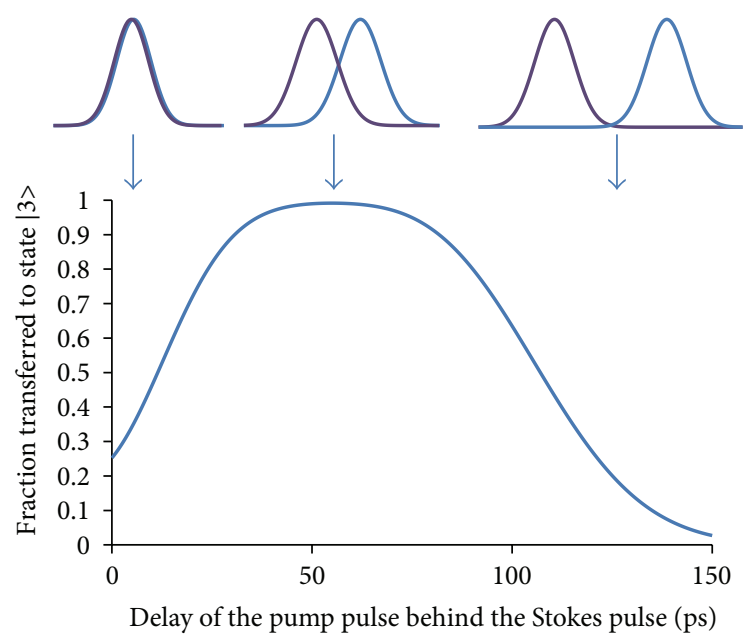

FIGURE 7: Fraction of molecules transferred into state $|3\rangle$ as a function of the delay between the pump and Stokes pulses. This calculation was performed with Gaussian laser pulses with a full width at half $\max =62 \mathrm{ps}$ and $\Omega_{p}=\Omega_{s}=0.213 \times 10^{12} \mathrm{rad} / \mathrm{s}$ (area $=$ 20 radians). The insets at the top indicate the relative timing of the pulses. The violet curve represents $\Omega_{s}$. The blue curve represents $\Omega_{p}$.

the maximum measurable STIRAP efficiency is slightly more than a factor of two greater than the SEP transfer efficiency when a grating-based spectrograph is used to collect the fluorescence. This rather small gain in signal is because the spectrograph collects light at the center of the lasers where the STIRAP transfer efficiency is $100 \%$ as well as the outer edges of the laser pulses where the pulses are much weaker. In other publications we demonstrate STIRAP transfer efficiencies that are more than a factor of two larger than the SEP transfer efficiency. Details of the work can be found in $[58,59]$.

We have also demonstrated STIRAP on target atoms in a buffer gas at atmospheric pressure. This was done on sodium vapor in an argon buffer gas. Graphs similar to Figure 8 were

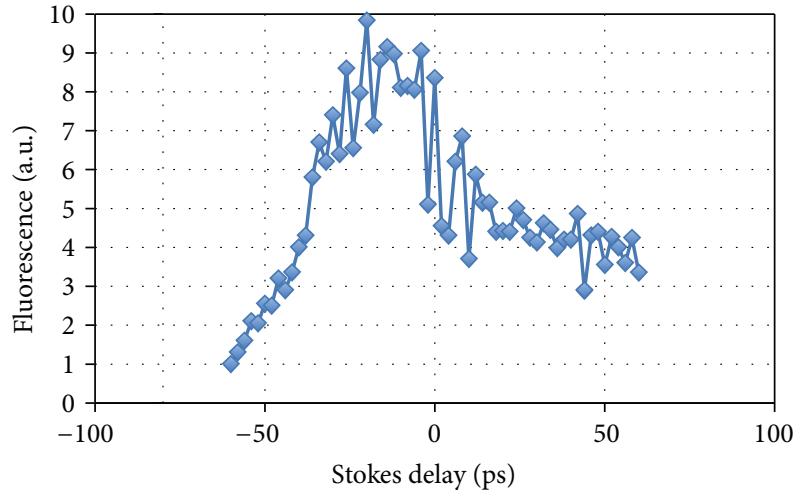

FIGURE 8: STIRAP on sodium vapor. A Stokes delay of 0 corresponds to perfect temporal overlap of the pump and Stokes pulses. Positive delays (pump before Stokes) correspond to SEP; negative delays (Stokes before pump) correspond to STIRAP.

collected at several argon pressures up to atmospheric pressure. From each of these graphs, the STIRAP signal (highest at negative Stokes delay) and SEP signal (measured at positive Stokes delay toward the right of the figure) were determined and a STIRAP/SEP ratio was calculated. In Figure 8 this ratio is approximately equal to 2 . Figure 9 shows this ratio as a function of argon pressure. If collisions were to interfere with the STIRAP process, the STIRAP/SEP ratio should drop as the argon pressure increased. The figure shows that there is no drop in the STIRAP transfer efficiency even up to atmospheric pressure. Additional details can be found in [60].

Such experiments represent not only the first picosecond STIRAP measurements, but also the first measurements on target species in a buffer gas beyond a 1 torr sample gas pressure. The total standoff distance for these measurements was less $<1 \mathrm{~m}$. However, with larger optical collection devices, which are available commercially, we believe such detection 


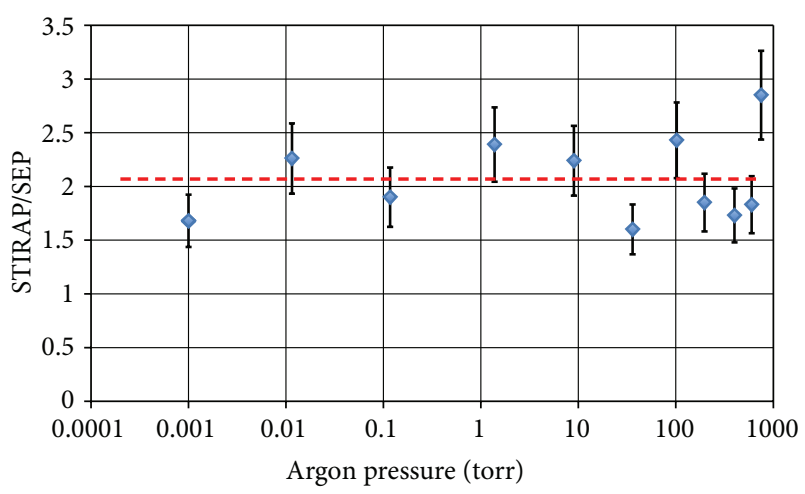

FIGURE 9: STIRAP/SEP ratio of sodium vapor as a function of argon buffer gas pressure.

distances can be easily extended to $50 \mathrm{~m}$. Certainly, such standoff capability has been achieved with other Raman methods [61]. A major hurdle from our perspective was achieving STIRAP at ambient conditions, that is, atmospheric pressures and room temperature. Finally, once a significant standoff capability has been realized, this method should prove invaluable in numerous applications from military defense related problems to environmental monitoring.

\section{Infrared Enhanced Dual Pulse LIBS}

Laser-induced breakdown spectroscopy (LIBS) provides sensitive, multi-elemental, and real time chemical analysis [62]. Because of its rapidity, small sample size, noncontact nature and freedom from sample preparation, LIBS has become a common technique for elemental analysis in a variety of applications, including analysis of rocks, art work, quality control in metal alloys and examination of the surface oxidation of inorganic and organic materials. The technique employs an intense, short duration laser pulse to ablate the sample, generating a rapidly evolving (ns- $\mu$ s scale) plasma. The ablation parameters are highly sample dependent and the resulting plasma plume is a transient, emissive mixture of atoms, ions and molecules in various states of excitation. In addition to evolving rapidly in time, the plasma is spatially inhomogeneous in composition and temperature. The plasma's thermal emission is collected and analyzed, typically with CCD or ICCD-based spectrographs. Relative elemental limits of detection are typically in the tens to low hundreds of ppm [63]. LIBS is ideally suited for remote sensing and hazardous materials applications because of its ease of use and because it requires little or no sample preparation. Nonetheless, the technique is limited by matrix effects and poor precision [64], in part because there is no sample preparation.

Although the first mention of DP LIBS (dual-pulse LIBS) for elemental analysis came from Malmstadt in 1969 [65], it has experienced a resurgence recently [66-72]. The technique provides enhanced LIBS signal via modification of the sample, atmosphere or plasma through the addition of a second laser pulse. DP LIBS pulse separations are typically on the order of a few microseconds, though separations may be as short as a few picoseconds in the case of femtosecond pulses. Relative pulse geometries are classified as either collinear or orthogonal, with the former lending itself to standoff work [73-77]. The DP LIBS technique has demonstrated signal enhancements of approximately an order of magnitude in some cases, though the values vary depending on the substrate and the particular element being investigated. Standoff applications frequently employ a laser pre-spark to generate a rarefied environment into which a subsequent analytical plasma can expand with minimal interference from the rarefied atmosphere (see [78]). The largest improvements have been obtained on pure metal substrates, while other substrates give enhancements that are less impressive.

The enhancement mechanisms of DP LIBS have been postulated to involve: (1) direct coupling of the second pulse into the plasma created by the first pulse (i.e., plasma "reheating"), (2) target heating by the first pulse, thus allowing enhanced ablation by the second pulse and higher resulting plasma temperatures and (3) formation of a thermally hot, low number density atmosphere above the sample surface by the first pulse, thereby altering the development and propagation of the analytical plasma created by the second pulse [31]. The latter scenario is particularly interesting due to the improved spectral line shape, improved signal to background ratio, and slower line shape and intensity evolution observed by Colao et al. [79]. Because of the reduced shielding, DP LIBS may also increase the ablation efficiency and the reproducibility of the analytical plasma. The dual-pulse phenomenon is of interest to the standoff laser sensing community as signal is typically at a premium in this geometry; signal collection falls off with the square of the standoff distance necessitating either larger optics or more signal spectral radiance. Note, however, that the exact relationship between the detection limit and standoff distance is also a function of the noise source and type.

Standoff LIBS, as well as DP LIBS have been investigated for the detection of explosives. For example, Gottfried et al. coupled PLS-DA (partial least squares discriminant analysis) and DP LIBS at standoff distances of $25 \mathrm{~m}$ to discriminate RDX $\geq 96 \%$ of the time on a variety of substrates (travertine and wood surfaces gave lower discrimination $-40 \%$ and $88 \%$, resp.) [80]. The discrimination was based on the ratios of 83 atomic and molecular emission lines. Even more recently, Waterbury et al. demonstrated infrared enhanced standoff detection of explosives at tens of meters using modest telescope optics [81].

As energetic materials are typically organic in nature, their decomposition byproducts are simple, light, and very stable molecules $\left(\mathrm{N}_{2}, \mathrm{H}_{2} \mathrm{O}, \mathrm{CO}, \mathrm{CO}_{2}\right)$. In addition to $\mathrm{C}, \mathrm{H}, \mathrm{O}$ and $\mathrm{N}$ atomic emission, the laser ablation of energetic materials also generates molecular species such as $\mathrm{CN}$ and $\mathrm{C}_{2}$ observable by optical emission spectroscopy. Because of air entrainment in the plasma and subsequent chemical reactions, it is difficult to distinguish between strictly samplederived $\mathrm{C}, \mathrm{N}, \mathrm{O}$, or $\mathrm{CN}$ emission and that resulting from the incorporation of air in the plasma and subsequent chemical reactions between air and sample-derived species. In the laboratory, air is excluded by flushing with a noble gas, while 


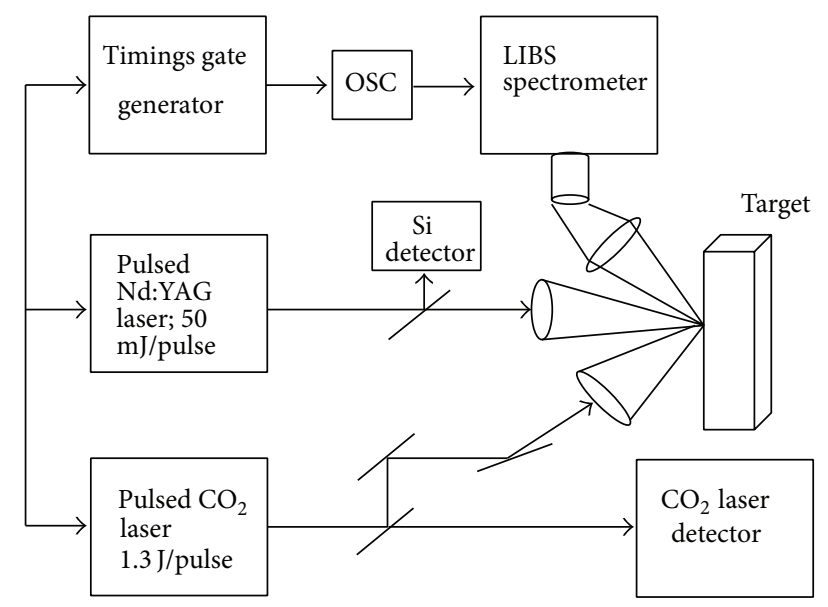

FIgURE 10: Schematic of the two laser LIBS systems for $\mathrm{CO}_{2}$ laser enhancement of Nd:YAG induced LIBS plasma emission for the preliminary work.

standoff applications profit from DP LIBS configurations where a prespark can reduce the number density of the surrounding atmosphere. A general understanding of the origin of the organic molecule-derived signals is crucial if these are to be used for explosive identification [82-85].

Our lab has investigated DP LIBS for potential to enhance standoff detection of explosives. Though Nd:YAG wavelengths dominate the dual-pulse literature, other laser combinations have been employed. Our research has focused on the use of a secondary $\mathrm{CO}_{2}$ laser pulse to reheat and prolong the plasma produced by an initial Nd:YAG-induced spark. It is well known from the laser cutting and welding industry that a $10.6 \mu \mathrm{m}$ laser couples very effectively into the lasergenerated plasma, creating "plasma shielding" of the target. Thus, the $\mathrm{CO}_{2}$ laser should couple into the plasma's free electrons through inverse bremsstrahlung. Although this DP LIBS configuration would not benefit from atmospheric exclusion, the increased signal may improve standoff detection. Of course, selectivity is also important to explosives identification, but this is beyond the scope of our initial work.

Preliminary $1.06 \mu \mathrm{m}+10.6 \mu \mathrm{m}$ DP LIBS experiments were reported by Killinger, Allen, and coworkers in 2007 using an alumina target with $\mathrm{Na}, \mathrm{Mg}$, Fe and $\mathrm{Si}$ impurities ( $\leq 0.08 \%$ as oxides) [86]. The experimental arrangement is given in Figure 10.

Results for aluminum and magnesium from this preliminary study are given in Figure 11. For the single pulse experiment, a Q-switched Nd:YAG (Big Sky CRF200, 50 mJ/pulse, $5 \mathrm{~ns}, 5 \mathrm{~Hz}, M^{2}=5$ ) was focused to a $1 \mathrm{~mm}$ diameter spot on the alumina target. Plasma emission was focused onto a $300 \mu \mathrm{m}$ inner core fiber optic cable coupled to a spectrograph (Ocean Optics LIBS2500, 200-980 nm). In the dual-pulsed experiments, a high power, $\mathrm{CO}_{2}$ TEA laser beam (Lumonics Model 920, $1.3 \mathrm{~J}, 5 \mathrm{~Hz}$ ) was fired at the alumina target in conjunction with the Nd:YAG laser. The laser operated at $10.6 \mu \mathrm{m}$ with a $100 \mathrm{~ns}$ spike followed by a $5 \mu$ s nitrogen-fed tail. The $\mathrm{CO}_{2}$ pulse was defocused to a fluence of $0.050 \mathrm{~J} / \mathrm{mm}^{2}$ on the target surface. A digital delay generator was used to time the pulses. A fast $\mathrm{Si}$ photodiode and photon drag detector were used to monitor the Nd:YAG and $\mathrm{CO}_{2}$ pulses, respectively. The angular separation of the Nd:YAG and $\mathrm{CO}_{2}$ beams was about 30 degrees, with the LIBS collection optics oriented at an angle of about 20 degrees to the $\mathrm{CO}_{2}$ beam as shown in Figure 10.

Several aluminum peaks are evident in the spectra in Figure 11. The strongest lines are around $394.40 \mathrm{~nm}$ and $396.15 \mathrm{~nm}$, although they are poorly resolved in the figure due to the low spectrometer resolution. The unlabeled peaks in other regions of the spectrum correspond to $\mathrm{Mg}, \mathrm{Na}$, and $\mathrm{H}$ impurities in the sample.

The infrared enhancement factor is defined here as the ratio of the peak heights for the enhanced signal (Figure 11) to the corresponding peak heights for the Nd:YAG LIBS signal (Figure 11) after subtracting the remaining background continuum. Although this ratio is only a crude estimate of the true gain that can be realized, it is useful for demonstrating trends in the signal enhancement. This factor is 5 to 20 for emission from atomic species while it is approximately 200 for ions. Neutral atomic atmospheric components have large signal enhancements because their spectrum is very weak for standard Nd:YAG LIBS, so any increase with the infrared laser creates a substantial enhancement factor. Several additional comments should be made about the experimental set-up: (1) No LIBS signal or visible plasma was observed with the $\mathrm{CO}_{2}$ laser alone, that is, the $\mathrm{CO}_{2}$ intensity was below the plasma breakdown threshold and (2) the collection time for the spectrometer was several ms so that significant atmospheric species were excited and detected. Given that the second pulse was $10.6 \mu \mathrm{m}$, the enhancement mechanism here is best understood as a continuation of the plasma by the second laser pulse rather than a reignition as discussed in Pershin [67]. It is also interesting to note that no enhancement occurred when the shorter Nd:YAG pulse occurred in the midst of the much longer $\mathrm{CO}_{2}$ pulse, perhaps suggesting decreased ablation efficiency as a result of the onset of plasma shielding and laser supported detonation when the Nd:YAG ablates the sample in the bath of mid-IR irradiance.

Subsequent work in our laboratory has focused on the benefits of the infrared reheating pulse for molecular emission measurements in LIBS plasmas. Although it is generally assumed that the primary source of $\mathrm{CN}$ species in the LIBS plasma subsequent to the ablation of organics is chemical reactions with air [78], it has been suggested that UV or femtosecond ablation creates diatomic molecules directly, potentially maintaining the information about bond structures in the original molecule $[83,84]$. Presumably, as the plasma evolves, more non-native $\mathrm{CN}$ is created through atmospheric reactions. Ma and Dagdigian have modeled the process, tracking molecular and atomic populations and their excitation state distributions [85]. Several groups conclude that emission from native $\mathrm{CN}$ bonds created by "selective" photolysis of chemical bonds in the ablation process should give rise to a single exponential-type decay of $\mathrm{CN}$ intensity, while recombination effects should lead to a more complex ingrowth over tens to hundreds of nanoseconds followed by a decay as the plasma cools and dissipates. Thus, at early times, it may be reasonable to assume that molecular emission originates from native molecules. 


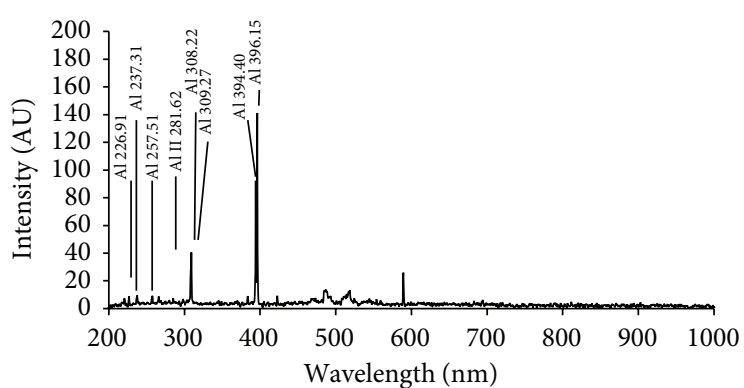

(a)

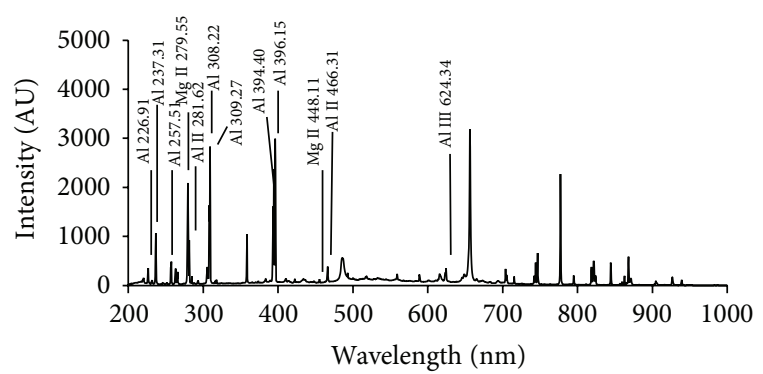

(b)

FIGURE 11: (a) LIBS signal from ceramic alumina target for Nd:YAG laser initiated plasma. (b) LIBS emission signal for a Nd:YAG laser initiated plasma and $\mathrm{CO}_{2}$ laser enhancement.

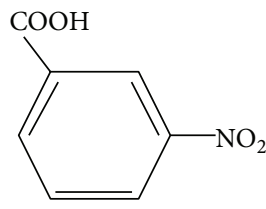

FIGURE 12: Structure of nitrobenzoic acid (NBA).

With this in mind, the benefit of infrared enhancement in early plasma molecular emission was investigated in a benchtop setting. Note that this work focuses on $\mathrm{S} / \mathrm{N}$ rather than selectivity as the former is simpler to quantify. Long-pulse $\mathrm{CO}_{2}$ lasers are not appropriate for enhancing molecular emission, as most of the energy couples to the plasma at times where non-native emission would dominate. Thus, a $40 \mathrm{~mJ}$ TEA $\mathrm{CO}_{2}$ laser (Edinburgh Instruments) with an $~ 50 \mathrm{~ns}$ pulse width was used for the infrared reheating pulse. Pressed pellets of nitrobenzoic acid sample served as a convenient molecular stand in for TNT (Figure 12). Primary ablation was with $5 \mathrm{~mJ}$ of $355 \mathrm{~nm}$ from a Quantel YG981E. The modest UV energy was used to keep the laser flux at the sample low $\left(0.6 \mathrm{~J} / \mathrm{cm}^{2}\right) .40 \mathrm{~mJ}$ of $10.6 \mathrm{~nm} \mathrm{CO}_{2}$ was brought in at $70 \mathrm{~ns}$, with a spot size of $\sim 1.5 \mathrm{~mm}$, creating a flux of $0.02 \mathrm{~J} / \mathrm{cm}^{2}$. CN violet band signal to background ratios were measured as a function of time with a gated ICCD (Andor iStar, $50 \mathrm{~ns}$ gate width) coupled to a $0.5 \mathrm{~m}$ Czerny-Turner spectrometer (Andor Shamrock).

Although S/B is not a perfect stand-in for signal to noise ratio without knowledge of the background noise character, Figure 13 suggests that the reheating due to the infrared pulse likely decreases the ability to perceive the molecular emission at early times. Plasma imaging suggests that the infrared pulse couples into the plasma efficiently in this arrangement (Figure 14). Once again, this is likely an inverse bremsstrahlung process that directly heats the electrons responsible for the LIBS continuum background. Note that the images are independently scaled - that is, absolute intensities cannot be inferred, but relative morphology can. Given that the increase in brightness is at the edge of the plasma, it is likely that the reheating pulse has a low penetration into the plasma, with the heat diffusing throughout the plasma subsequently. Comparison of Figures 14 and 15 suggests that

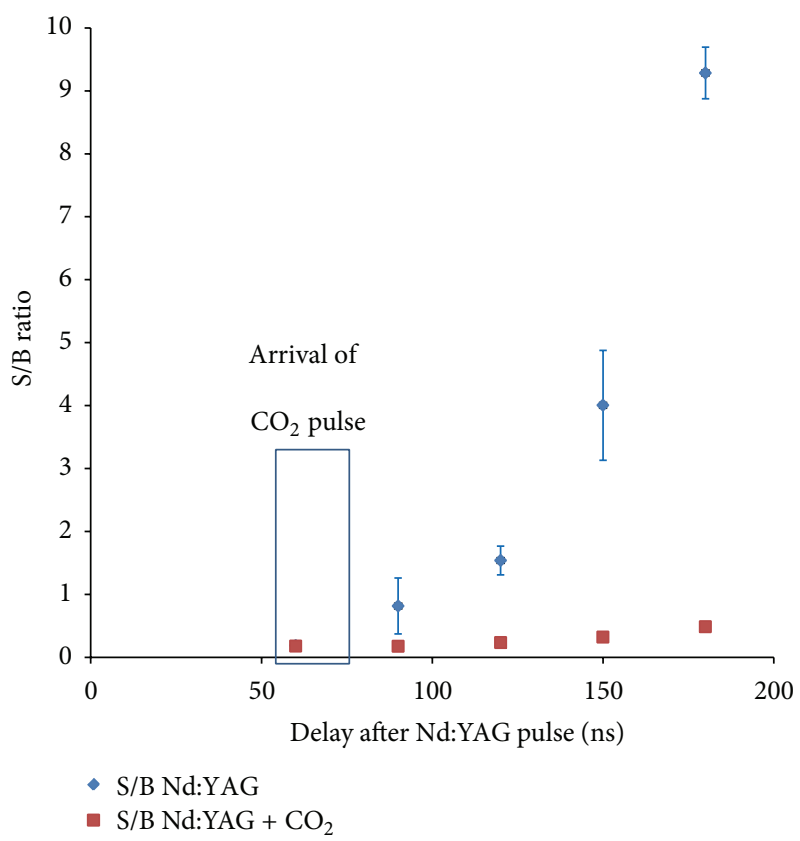

FIGURE 13: Effect of short-pulse $\mathrm{CO}_{2}$ reheating pulse on CN $\Delta v=0$ signal to background ratio.

the extent of the infrared energy's diffusion is actually fairly limited, at least on this brief timescale.

In light of these data, we would not expect $\mathrm{CO}_{2}$ laser reheating to improve native molecule-based detection and discrimination of explosives. It should be noted that the arrangement used with our double pulse work is unique in that we reheat with a mid IR pulse and we couple the reheating pulse in with only a $100 \mathrm{~ns}$ delay. The latter is dictated by our interest in native molecular signals. While we have observed enhancement of atomic signals using long-pulse $\mathrm{CO}_{2}$ TEPS and portable spectrometers, this measured enhancement should be qualified. First, the enhancement is most significant for those species that benefit most from a more energetic plasma (e.g., ionic species). Second, enhancement of the signal does not necessarily improve detection as noise "enhancement factor" could match or exceed the signal enhancement if plasma 


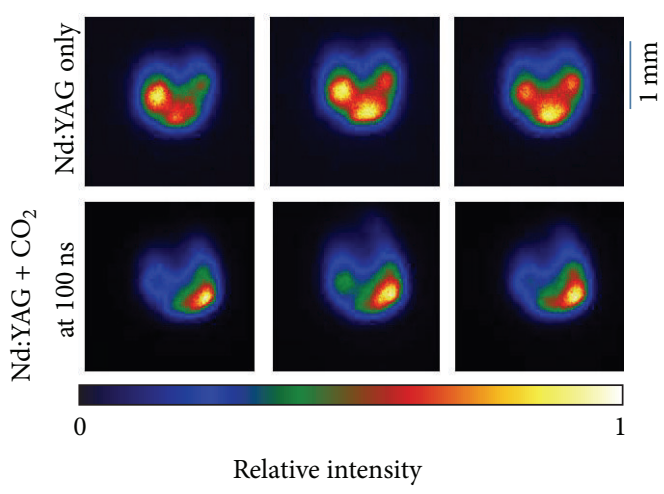

FIGURE 14: Effect of reheating $\mathrm{CO}_{2}$ pulse on spectrally integrated plasma images. The reheating laser impinges on the plasma from the lower right in this camera angle. The target is at the top of each image. The $\mathrm{CO}_{2}$ pulse arrives $100 \mathrm{~ns}$ after the Nd:YAG ablation pulse, with the ICCD gate set to $180 \mathrm{~ns}$ after the Nd:YAG pulse.

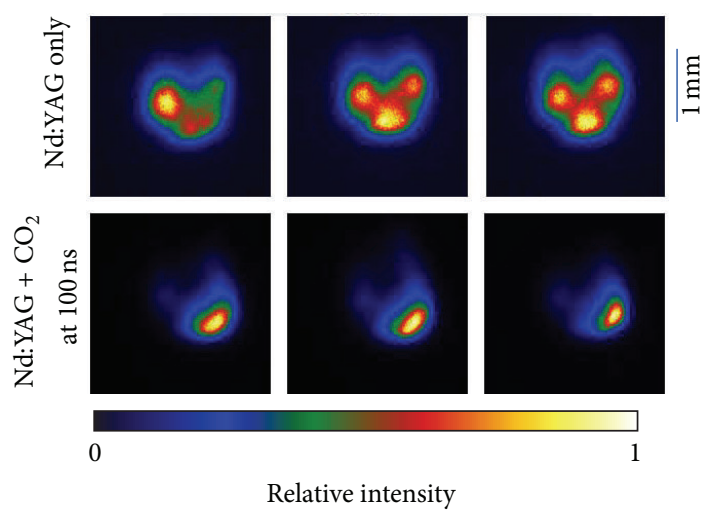

Figure 15: As Figure 14, but with 210 ns imaging delay.

continuum (i.e., bremsstrahlung and recombination emission) is the limiting noise, as is commonly the case in LIBS measurements, especially when using intensified detectors. Finally, as the LIBS plasma size and time evolution is likely different between single pulse and $\mathrm{CO}_{2}$-reheated plasmas [87], the analytical performance of each technique should be optimized separately before comparison. Such a full comparison is quite complex and beyond the scope of this initial report. The use of $\mathrm{a} \mathrm{CO}_{2}$ laser pulse as a preparatory prespark (in contrast to the reheating described here) pulse may also be of interest. It remains to be seen if a $\mathrm{CO}_{2}$ prespark can create a low number-density atmosphere for the subsequent analytical plasma; this concept is currently being investigated and will be addressed in future publications.

\section{Conclusion}

Two promising techniques for standoff detection have been reviewed and discussed. One method, STIRAP, has been limited in the past to species in molecular beams or subatmospheric gas samples (low-pressure bulk gases). However that method, at least in the picosecond time regime, has now been successfully demonstrated on gas samples at atmospheric pressures. Although STIRAP has yet to be demonstrated on species associated with threat agents, one of the major hurdles, operation at atmospheric pressures, has been accomplished. Infrared enhanced dual pulse LIBS is further along in that it is currently being used to detect explosives or, as discussed in this work, explosive simulants. However there is still a great deal of questions associated with the infrared enhancement and further studies will most likely be required before this method can achieve its full potential as a viable standoff detection method for explosives and other threat agents.

\section{Disclosure}

The material presented here has been approved as Distribution A: Unlimited Public Release.

\section{Conflict of Interests}

The authors declare that there is no conflict of interests regarding the publication of this paper.

\section{Acknowledgments}

The authors would like to acknowledge the efforts of the graduate students and postdocs from the Spectral Signatures, Multiphoton and LAMP laboratories within ArCLAS at Arkansas State University. This work was funded by the U.S. Army RDECOM CERDEC Night Vision and Electronic Sensors Directorate under Contract nos. \#W909MY-09-C0001 and \#W15P7T-10-C-A012.

\section{References}

[1] J. I. Steinfeld and J. Wormhoudt, "Explosives detection: a challenge for physical chemistry," Annual Review of Physical Chemistry, vol. 49, no. 1, pp. 203-232, 1998.

[2] D. S. Moore, "Instrumentation for trace detection of high explosives," Review of Scientific Instruments, vol. 75, no. 8, pp. 24992512,2004

[3] R. L. Woodfin, Ed., Trace Chemical Sensing of Explosives, John Wiley \& Sons, New York, NY, USA, 2006.

[4] C. A. Munson, J. L. Gottfried, F. C. de Lucia Jr., K. L. McNesby, and A. W. Miziolek, Laser-based detection methods for explosives, ARL-TR-4279, 2007.

[5] S. Wallin, A. Pettersson, H. Östmark, and A. Hobro, "Laserbased standoff detection of explosives: a critical review," Analytical and Bioanalytical Chemistry, vol. 395, no. 2, pp. 259-274, 2009.

[6] L. A. Skvortsov, "Laser methods for detecting explosive residues on surfaces of distant objects," Quantum Electronics, vol. 42, no. 1, pp. 1-11, 2012.

[7] J. Wormhoudt, J. H. Shorter, J. B. McManus et al., “Tunable infrared laser detection of pyrolysis products of explosives in soils," Applied Optics, vol. 35, no. 21, pp. 3992-3997, 1996.

[8] M. W. Todd, R. A. Provencal, T. G. Owano et al., "Application of mid-infrared cavity-ringdown spectroscopy to trace explosives vapor detection using a broadly tunable $(6-8 \mu \mathrm{m})$ optical parametric oscillator," Applied Physics B: Lasers and Optics, vol. 75, no. 2-3, pp. 367-376, 2002. 
[9] D. Heflinger, T. Arusi-Parpar, Y. Ron, and R. Lavi, "Application of a unique scheme for remote detection of explosives," Optics Communications, vol. 204, no. 1-6, pp. 327-331, 2002.

[10] T. M. Swager, "Polymer electronics for explosives detection," in Electronic Noses \& Sensors for the Detection of Explosives, J. W. Gardner and J. Yinon, Eds., vol. 159 of NATO Science Series II: Mathematics, Physics and Chemistry, pp. 29-37, Kluwer Academic Publishers, New York, NY, USA, 2004.

[11] C. Cumming, M. Fisher, and J. Sikes, "Amplifying fluorescent polymer arrays for chemical detection of explosives," in Electronic Noses \& Sensors for the Detection of Explosives, W. Gardner and J. Yinon, Eds., vol. 159 of NATO Science Series II: Mathematics, Physics and Chemistry, pp. 53-69, Kluwer Academic Publishers, Dordrecht, The Netherlands, 2004.

[12] M. Fisher and J. Sikes, "Detection of landmines and other explosives with an ultra-trace chemical detector," in Electronic Noses \& Sensors for the Detection of Explosives, J.W. Gardner and J. Yinon, Eds., vol. 159 of NATO Science Series II: Mathematics, Physics and Chemistry, pp. 117-130, Kluwer Academic Publishers, Dodrecht, The Netherlands, 2004.

[13] M. B. Pushkarsky, I. G. Dunayevskiy, M. Prasanna, A. G. Tsekoun, R. Go, and C. K. N. Patel, "High-sensitivity detection of TNT," Proceedings of the National Academy of Sciences of the United States of America, vol. 103, no. 52, pp. 19630-19634, 2006.

[14] C. A. Kendziora, R. Furstenberg, M. Papantonakis et al., "Infrared photothermal imaging of trace explosives on relevant substrates," in Detection and Sensing of Mines, Explosive Objects, and Obscured Targets, vol. 8709 of Proceedings of SPIE, Baltimore, Maryland, USA, 2013.

[15] C. M. Wynn, S. Palmacci, R. R. Kunz, and M. Aernecke, "Noncontact optical detection of explosive particles via photodissociation followed by laserinduced fluorescence," Optics Express, vol. 19, no. 19, pp. 18671-18677, 2011.

[16] J. J. Brady and P. M. Pellegrino, "Next generation hazard detection via ultrafast coherent anti-Stokes Raman spectroscopy," in Chemical, Biological, Radiological, Nuclear, and Explosives (CBRNE) Sensing XIV, 87100Q, vol. 8710 of Proceedings of SPIE, Baltimore, Md, USA, April 2013.

[17] C. W. Van Neste, L. R. Senesac, and T. Thundat, "Standoff spectroscopy of surface adsorbed chemicals," Analytical Chemistry, vol. 81, no. 5, pp. 1952-1956, 2009.

[18] J. Hildenbrand, J. Herbst, J. W, A. Lambrecht, and J. Wöllenstein, "Explosive detection using infrared laser spectroscopy," in Proceedings of the SPIE Quantum Sensing and Nanophotonic Devices VI, vol. 7222, 2009.

[19] C. Eliasson, N. A. Macleod, and P. Matousek, "Non-invasive detection of concealed liquid explosives using Raman spectroscop," Analytical Chemistry, vol. 79, pp. 8146-8152, 2007.

[20] "Cavity Ringdown Spectroscopy: an ultratrace absorption measurement technique," in Proceedings of the ACS Symposium Series 720, K. W. Busch and M. A. Busch, Eds., American Chemical Society, Washington, DC, USA, 1999.

[21] G. Berden and R. Engelin, Eds., Cavity Ringdown Spectroscopy, John Wiley \& Sons, Chichester, UK, 2009.

[22] A. W. Miziolek, V. Palleschi, and I. Schechter, Eds., History and Fundamentals of LIBS, Cambridge University Press, 2006.

[23] R. S. Harmon, F. C. De Lucia, A. W. Miziolek, K. L. McNesby, R. A. Walters, and P. D. French, "Laser-induced breakdown spectroscopy (LIBS) - an emerging field-portable sensor technology for real-time, in-situ geochemical and environmental analysis," Geochemistry: Exploration, Environment, Analysis, vol. 5, no. 1, pp. 21-28, 2005.
[24] T. F. Jenkins, D. C. Leggett, and T. A. Ranney, Vapor Signatures from Military Explosives, Special Report no. 99-21, US Army Corps of Engineers, 1999.

[25] A. Goth, I. G. McLean, and J. Trevelyan, "How do dogs detect landmines? A summary of research results," in Mine Detection Dogs: Training, Operations, and Odour Detection, I. G. McLean, Ed., pp. 195-208, GICHD, Geneva, Switzerland, 2003.

[26] M. Krausa, "Vapor detection of explosives from counter-terrorism," in Vapour and Trace Detection of Explosives for AntiTerrorism Purposes, vol. 167 of NATO Science Series II: Mathematics, Physics and Chemistry, pp. 1-9, Springer, Dordrecht, The Netherlands, 2004.

[27] M. Stancl, "Detection of traces of explosives by means of sniffing dogs," in Vapor and Trace Detection of Explosives for AntiTerrorism Purposes, vol. 167 of NATO Sciences Series II: Mathematics, Physics, and Chemistry, pp. 66-77, 2004.

[28] J. D. Pleil, "Role of exhaled breath biomarkers in environmental health science," Journal of Toxicology and Environmental Health B: Critical Reviews, vol. 11, no. 8, pp. 613-629, 2008.

[29] J. H. Raymer, E. D. Pellizzarri, K. W. Thomas, and S. D. Cooper, "Elimination of volatile organics in breath after exposure to occupational and environmental microenvironments," Journal of Exposure Analysis and Environmental Epidemiology, vol. 1, pp. 439-451, 1991.

[30] S. Schiemann, A. Kuhn, S. Steuerwald, and K. Bergmann, "Efficient coherent population transfer in NO molecules using pulsed lasers," Physical Review Letters, vol. 71, no. 22, pp. 36373640, 1993.

[31] D. A. Cremers and L. J. Radziemski, "History and fundamentals of LIBS," in Laser-Induced Breakdown Spectroscopy (LIBS): Fundamentals and Applications, A. W. Miziolek, V. Palleschi, and I. Schechter, Eds., Cambridge Univerity Press, Cambridge, UK, 2006.

[32] L. S. Rothman, D. Jacquemart, A. Barbe et al., "The HITRAN 2004 molecular spectroscopic database," Journal of Quantitative Spectroscopy and Radiative Transfer, vol. 96, no. 2, pp. 139-204, 2005.

[33] L. S. Rothman, I. E. Gordon, A. Barbe et al., "The HITRAN 2008 molecular spectroscopic database," Journal of Quantitative Spectroscopy and Radiative Transfer, vol. 110, pp. 533-572, 2009.

[34] K. G. Furton and L. J. Myers, "The scientific foundation and efficacy of the use of canines as chemical detectors for explosives," Talanta, vol. 54, no. 3, pp. 487-500, 2001.

[35] "Compendium of Methods for the Determination of Toxic Organic Compounds in Ambient Air," 2nd Edition EPA/625/R96/010b, 1999.

[36] U.S. Environmental Protection Agency (EPA), Method-320, Measurement of Vapor Phase Organic and Inorganic Emissions by Extractive Fourier Transform Infrared Spectroscopy, 1999.

[37] L. R. Brown, M. R. Gunson, R. A. Toth, F. W. Irion, C. P. Rinsland, and A. Goldman, "1995 atmospheric trace molecule spectroscopy (ATMOS) linelist," Applied Optics, vol. 35, no. 16, pp. 2828-2848, 1996.

[38] N. Jacquinet-Husson, E. Arié, J. Ballard et al., “The 1997 spectroscopic GEISA databank," Journal of Quantitative Spectroscopy and Radiative Transfer, vol. 62, pp. 205-254, 1999.

[39] N. Husson, B. Bonnet, N. A. Scott, and A. Chedin, "Management and study of spectroscopic information: the GEISA program," Journal of Quantitative Spectroscopy and Radiative Transfer, vol. 48, no. 5-6, pp. 509-518, 1992. 
[40] L. S. Rothman, C. P. Rinsland, A. Goldman et al., "The Hitran molecular spectroscopic database and Hawks (Hitran atmospheric workstation): 1996 edition," Journal of Quantitative Spectroscopy and Radiative Transfer, vol. 60, no. 5, pp. 665-710, 1998.

[41] Midac Corporation, Standards: Gas Phase Infrared Spectral Standards, A collection of approximately 150, vapor phase, infrared spectra supplied in digital format. Midac Corporation, Irvine, California, USA, http://www.midac.com/.

[42] P. Hanst, "A collection of 296 vapor phase infrared spectra supplied in digital format," Infrared Spectra for Quantitative Analysis of Gases, Infrared Analysis, Anaheim, Calif, USA, http://www.infrared-analysis.com/.

[43] "NIST/EPA Gas-Phase Infrared Database," World Wide Web, http://www.nist.gov/mml/csd/informatics_research/webbook chemident.cfm.

[44] C. J. Pouchert, Ed., The Aldrich Library of FT-IR Spectra Vapor Phase, vol. 3, The Aldrich Chemical, Milwaukee, Wis, USA, 1st edition, 1989.

[45] S. W. Sharpe, T. J. Johnson, R. L. Sams, P. M. Chu, G. C. Rhoderick, and P. A. Johnson, "Gas-phase databases for quantitative infrared spectroscopy," Applied Spectroscopy, vol. 58, no. 12, pp. 1452-1461, 2004.

[46] T. J. Johnson, R. L. Sams, S. D. Burton, and T. A. Blake, "Absolute integrated intensities of vapor-phase hydrogen peroxide $\left(\mathrm{H}_{2} \mathrm{O}_{2}\right)$ in the mid-infrared at atmospheric pressure," Analytical and Bioanalytical Chemistry, vol. 395, no. 2, pp. 377-386, 2009.

[47] T. Osborn, W. A. Burns, J. Green, and S. W. Reeve, "An optical nose approach to explosive detection: one strategy for optically based sensing," Spectroscopy, vol. 26, no. 1, pp. 34-45, 2011.

[48] C. J. Lue, M. N. Sullivan, M. Draganjac, and S. W. Reeve, "Cavity ringdown spectroscopy of ${ }^{13} \mathrm{C}_{2} \mathrm{H}_{2}$ in the $12900-13400 \mathrm{~cm}^{-1}$ region," Journal of Molecular Spectroscopy, vol. 273, no. 1, pp. 6$10,2012$.

[49] Z. Gutmann, T. N. Clasp, C. J. Lue et al., "Quantum cascade laser FM spectroscopy of explosives," in Chemical, Biological, Radiological, Nuclear, and Explosives (CBRNE) Sensing XIV, vol. 8710 of Proceedings of SPIE, 87000J1-8, Baltimore, Md, USA, May 2013.

[50] T. N. Clasp and S. W. Reeve, "FTIR and diode laser spectroscopy of isobutylene: analysis of the rotational structure in the v28 fundamental band," Journal of Molecular Spectroscopy, vol. 266, no. 2, pp. 92-98, 2011.

[51] T. Osborn, Infrared sensing of isobutylene [M.S. thesis], 2012.

[52] L. H. Laurie and V. W. Scharpen, "Microwave spectroscopy of isobutylene," Analytical Chemistry, vol. 44, no. 5, pp. 378-384, 1972.

[53] Z. Gutmann, Rovibrational analysis of the Nu7 band of isobutylene [M.S. thesis], 2014.

[54] U. Gaubatz, P. Rudecki, S. Schiemann, and K. Bergmann, "Population transfer between molecular vibrational levels by stimulated Raman scattering with partially overlapping laserfields. A new concept and experimental results," The Journal of Chemical Physics, vol. 92, no. 9, pp. 5363-5376, 1990.

[55] T. Halfmann and K. Bergmann, "Coherent population transfer and dark resonances in $\mathrm{SO}_{2}$," Journal of Chemical Physics, vol. 104, no. 18, pp. 7068-7072, 1996.

[56] B. Broers, H. B. van Linden van den Heuvell, and L. D. Noordam, "Efficient population transfer in a three-level ladder system by frequency-swept ultrashort laser pulses," Physical Review Letters, vol. 69, no. 14, pp. 2062-2065, 1992.
[57] P. Pillet, C. Valentin, R.-L. Yuan, and J. Yu, "Adiabatic population transfer in a multilevel system," Physical Review A, vol. 48, no. 1, pp. 845-848, 1993.

[58] J. B. Johnson, K. Lyon, W. D. Murry, D. R. Britton, and M. J. Johnson, "Picosecond standoff multiphoton detection of gas phase species: initial results," in 4th Enabling Photonics Technologies for Defense, Security, and Aerospace Applications, vol. 6975 of Proceedings of SPIE, pp. 69750S/1-69750S/9, Orlando, Fla, USA, March 2008.

[59] J. B. Johnson, S. Allen, D. R. Britton et al., "Picosecond multiphoton STIRAP detection of gas phase species: a test with sodhim," in Proceedings of the Chemical, Biological, Radiological, Nuclear, and Explosives (CBRNE) Sensing X, April 2009.

[60] J. B. Johnson, S. D. Allen, J. L. Hicks, and J. Burdin, "STIRAP on sodium gas as a function of argon buffer gas pressure," in Chemical, Biological, Radiological, Nuclear, and Explosives (CBRNE) Sensing XI, vol. 7665 of Proceedings of the SPIE, Orlando, Fla, USA, April 2010.

[61] J. C. Carter, S. M. Angel, M. Lawrence-Snyder, J. Scaffidi, R. E. Whipple, and J. G. Reynolds, "Standoff detection of high explosive materials at 50 meters in ambient light conditions using a small Raman instrument," Applied Spectroscopy, vol. 59, no. 6, pp. 769-775, 2005.

[62] J. D. Winefordner, I. B. Gornushkin, T. Correll, E. Gibb, B. W. Smith, and N. Omenetto, "Comparing several atomic spectrometric methods to the super stars: special emphasis on laser induced breakdown spectrometry, LIBS, a future super star," Journal of Analytical Atomic Spectrometry, vol. 19, no. 9, pp. 1061-1083, 2004.

[63] R. S. Harmon, F. C. de Lucia, A. W. Miziolek, K. L. McNesby, R. A. Walters, and P. D. French, "Laser-induced breakdown spectroscopy (LIBS) - an emerging field-portable sensor technology for real-time, in-situ geochemical and environmental analysis," Geochemistry: Exploration, Environment, Analysis, vol. 5, no. 1, pp. 21-28, 2005.

[64] D. W. Hahn and N. Omenetto, "Laser-induced breakdown spectroscopy (LIBS) - II: review of instrumental and methodological approaches to material analysis and applications to different fields," Applied Spectroscopy, vol. 66, pp. 347-419, 2012.

[65] E. H. Piepmeier and H. V. Malmstadt, "Q-switched laser energy absorption in the plume of an aluminum alloy," Analytical Chemistry, vol. 41, no. 6, pp. 700-707, 1969.

[66] E. Tognoni and G. Cristoforetti, "Basic mechanisms of signal enhancement in ns double-pulse laser-induced breakdown spectroscopy in a gas environment," Journal of Analytical Atomic Spectrometry, 2014.

[67] S. M. Pershin, "Transformation of the optical spectrum of a laser plasma when a surface is irradiated with a double pulse," Soviet Journal of Quantum Electronics, vol. 19, no. 2, p. 215, 1989.

[68] J. Scaffidi, S. M. Angel, and D. A. Cremers, "Emission enhancement mechanisms in dual-pulse LIBS," Analytical Chemistry, vol. 78, no. 1, pp. 24-32, 2006.

[69] D. N. Stratis, K. L. Eland, and S. M. Angel, "Enhancement of aluminum, titanium, and iron in glass using pre-ablation spark dual-pulse LIBS," Applied Spectroscopy, vol. 54, no. 12, pp. 1719$1726,2000$.

[70] D. A. Cremers, L. J. Radziemski, and T. R. Loree, "Spectrochemical analysis of liquids using the laser spark," Applied Spectroscopy, vol. 38, no. 5, pp. 721-729, 1984.

[71] R. Nyga and W. Neu, "Double-pulse technique for optical emission spectroscopy of ablation plasmas of samples in liquids," Optics Letters, vol. 18, no. 9, pp. 747-749, 1993. 
[72] S. Nakamura, Y. Ito, K. Sone, H. Hiraga, and K. Kaneko, "Determination of an iron suspension in water by laser-induced breakdown spectroscopy with two sequential laser pulses," Analytical Chemistry, vol. 68, no. 17, pp. 2981-2986, 1996.

[73] C. Gautier, P. Fichet, D. Menut, J. L. Lacour, D. L'Hermite, and J. Dubessy, "Quantification of the intensity enhancements for the double-pulse laser-induced breakdown spectroscopy in the orthogonal beam geometry," Spectrochimica Acta B: Atomic Spectroscopy, vol. 60, no. 2, pp. 265-276, 2005.

[74] L. St-Onge, V. Detalle, and M. Sabsabi, "Enhanced laserinduced breakdown spectroscopy using the combination of fourth-harmonic and fundamental Nd:YAG laser pulses," Spectrochimica Acta B, vol. 57, no. 1, pp. 121-135, 2002.

[75] R. Sattmann, V. Sturm, and R. Noll, "Laser-induced breakdown spectroscopy of steel samples using multiple Q-switch Nd:YAG laser pulses," Journal of Physics D, vol. 28, no. 10, p. 2181, 1995.

[76] C. Gautier, P. Fichet, D. Menut, and J. Dubessy, "Applications of the double-pulse laser-induced breakdown spectroscopy (LIBS) in the collinear beam geometry to the elemental analysis of different materials," Spectrochimica Acta B: Atomic Spectroscopy, vol. 61, no. 2, pp. 210-219, 2006.

[77] J. Uebbing, J. Brust, W. Sdorra, F. Leis, and K. Niemax, "Reheating of a laser-produced plasma by a second pulse laser," Applied Spectroscopy, vol. 45, no. 9, pp. 1419-1423, 1991.

[78] J. L. Gottfried, F. C. de Lucia Jr., C. A. Munson, and A. W. Miziolek, "Laser-induced breakdown spectroscopy for detection of explosives residues: a review of recent advances, challenges, and future prospects," Analytical and Bioanalytical Chemistry, vol. 395, no. 2, pp. 283-300, 2009.

[79] F. Colao, V. Lazic, R. Fantoni, and S. Pershin, "A comparison of single and double pulse laser-induced breakdown spectroscopy of aluminum samples," Spectrochimica Acta B: Atomic Spectroscopy, vol. 57, no. 7, pp. 1167-1179, 2002.

[80] J. L. Gottfried, F. C. De Lucia Jr., and A. W. Miziolek, "Discrimination of explosive residues on organic and inorganic substrates using laser-induced breakdown spectroscopy," Journal of Analytical Atomic Spectrometry, vol. 24, no. 3, pp. 288-296, 2009.

[81] Alaka'i Consulting and Engineering, private communication, Largo, Fla, USA.

[82] P. Lucena, A. Doña, L. M. Tobaria, and J. J. Laserna, "New challenges and insights in the detection and spectral identification of organic explosives by laser induced breakdown spectroscopy," Spectrochimica Acta B, vol. 66, no. 1, pp. 12-20, 2011.

[83] M. Baudelet, L. Guyon, J. Yu et al., "Spectral signature of native $\mathrm{CN}$ bonds for bacterium detection and identification using femtosecond laser-induced breakdown spectroscopy," Applied Physics Letters, vol. 88, Article ID 063901, 2006.

[84] M. Boueri, M. Baudelet, J. Yu, X. Mao, S. S. Mao, and R. Russo, "Early stage expansion and time-resolved spectral emission of laser-induced plasma from polymer," Applied Surface Science, vol. 255, no. 24, pp. 9566-9571, 2009.

[85] Q. L. Ma and P. J. Dagdigian, "Kinetic model of atomic and molecular emissions in laser-induced breakdown spectroscopy of organic compounds," Analytical and Bioanalytical Chemistry, vol. 400, pp. 3193-3205, 2011.

[86] D. K. Killinger, S. D. Allen, R. D. Waterbury, C. Stefano, and E. L. Dottery, "Enhancement of Nd: YAG LIBS emission of a remote target using a simultaneous $\mathrm{CO}_{2}$ laser pulse," Optics Express, vol. 15, no. 20, pp. 12905-12915, 2007.
[87] A. De Giacomo, M. Dell'Aglio, D. Bruno, R. Gaudiuso, and O. De Pascale, "Experimental and theoretical comparison of single-pulse and double-pulse laser induced breakdown spectroscopy on metallic samples," Spectrochimica Acta B: Atomic Spectroscopy, vol. 63, no. 7, pp. 805-816, 2008. 

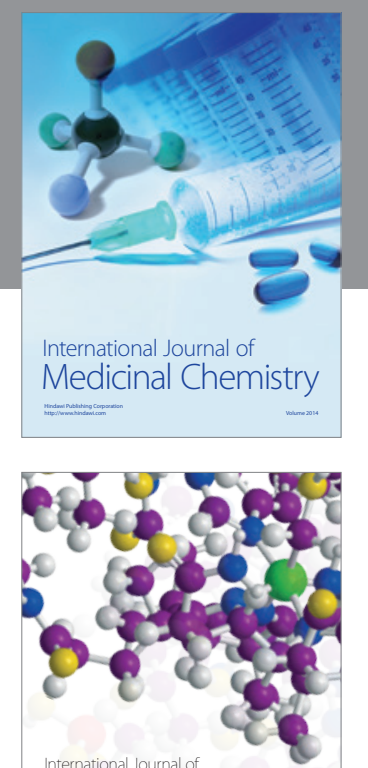

\section{Carbohydrate} Chemistry

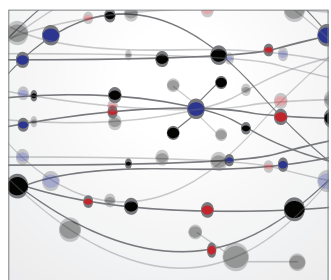

The Scientific World Journal
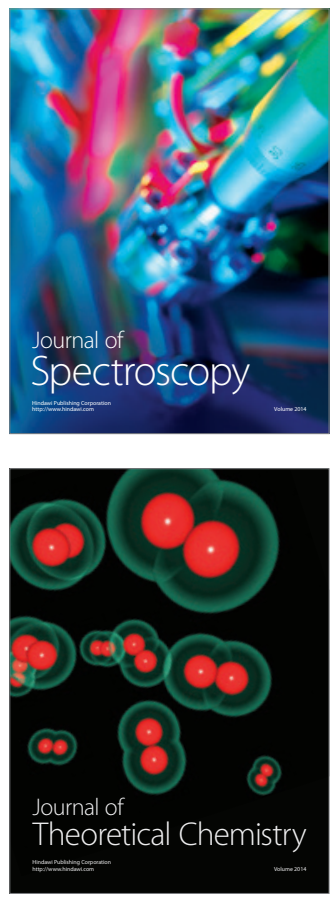
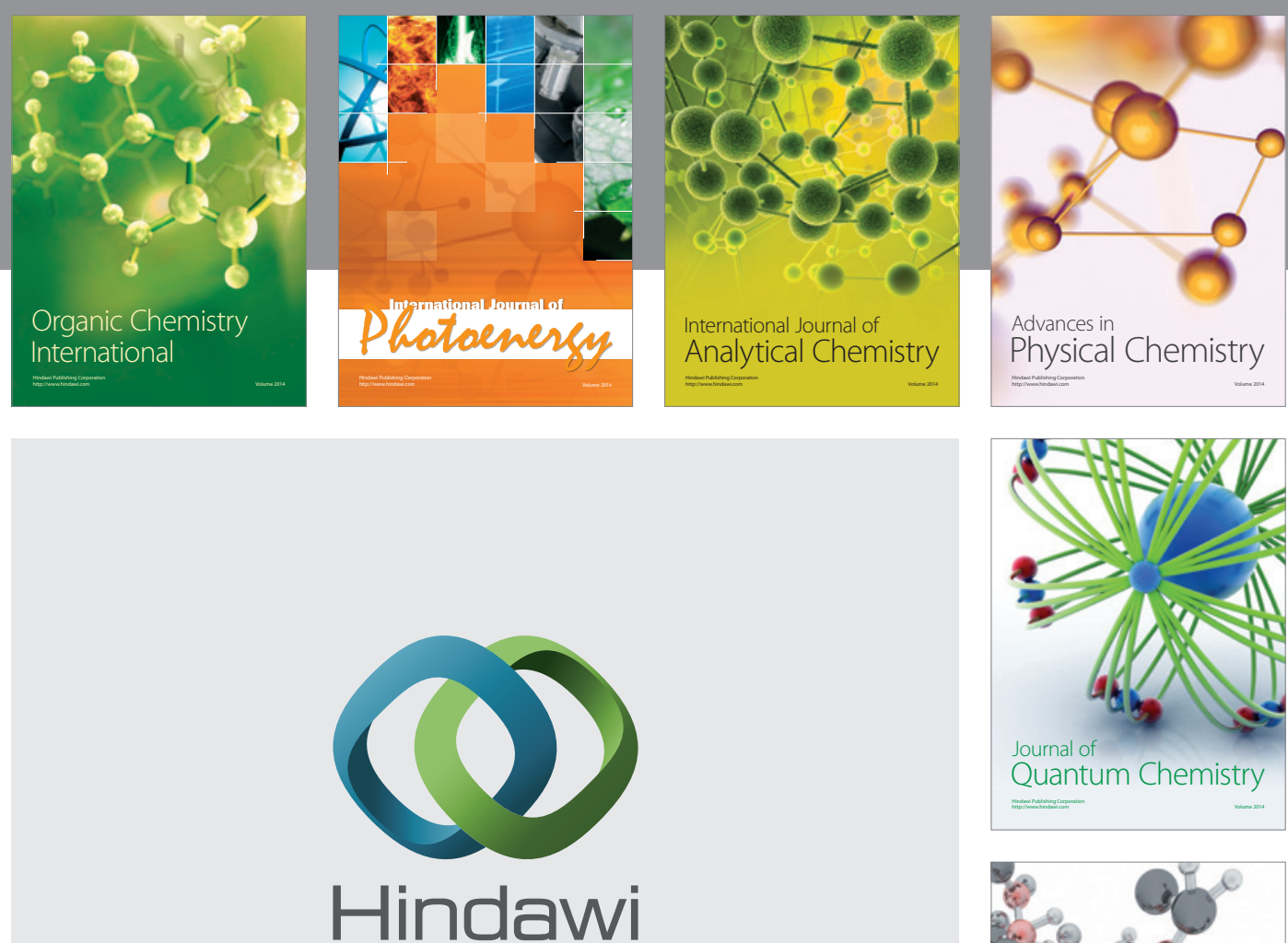

Submit your manuscripts at

http://www.hindawi.com

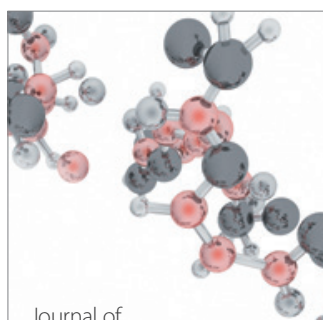

Analytical Methods

in Chemistry

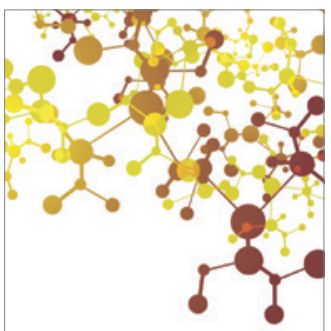

Journal of

Applied Chemistry

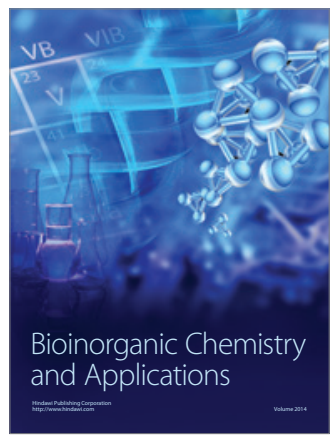

Inorganic Chemistry
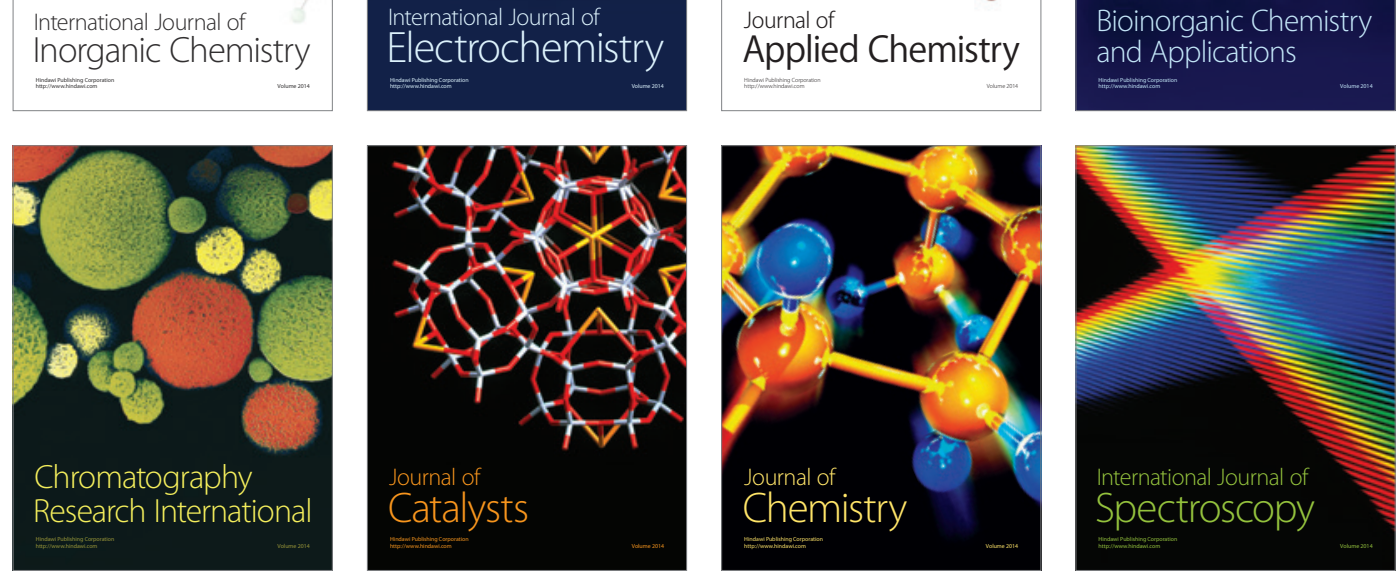